\title{
Buildup of Sn@CNT nanorods by in-situ thermal plasma and the electronic transport behaviors
}

\author{
Dongxing Wang ${ }^{1}, \mathrm{Da} \mathrm{Li}^{2}$, Javid. Muhammad ${ }^{1}$, Yuanliang Zhou ${ }^{1}$, Xuefeng Zhang ${ }^{3}$, Ziming Wang ${ }^{1}$, \\ Shanshan $\mathrm{Lu}^{1}$, Xinglong Dong ${ }^{1^{*}}$ and Zhidong Zhang ${ }^{2^{*}}$
}

\begin{abstract}
Monocrystal Sn nanorods encapsulated in the multi-walled carbon nanotubes (Sn@CNT NRs), were fabricated by a facile arc-discharge plasma process, using bulk $S n$ as the raw target and methane as the gaseous carbon source. The typical Sn@CNT NRs are 40-90 nm in diameter and 400-500 $\mathrm{nm}$ in length. The CNTs protect the inner Sn nanorods from oxidation. Temperature dependent $I-V$ curve and electronic resistance reveal that the dielectric behavior of Sn@CNT NRs is attributed to the multi-wall CNTs shell and follows Mott-David variable range hopping $\left[\ln R(T) \propto T^{-1 / 4}\right]$ model above the superconducting critical temperature of $3.69 \mathrm{~K}$, with semiconductor-superconductor transition (SST). Josephson junction of $\mathrm{Sn} / \mathrm{CNT} / \mathrm{Sn}$ layered structure is responsible for the superconducting behavior of Sn@CNT NRs.
\end{abstract}

Keywords: carbon nanotubes, nanocomposite, dielectric, variable range hopping, Josephson junction

\section{INTRODUCTION}

Encapsulation of nanostructured metals in graphite layers can protect the metallic core from oxidation. These carbon-coated nanostructures are now attracting more interest, due to their potential applications as functional materials such as lithium-ion batteries, fuel cell and electromagnetic wave absorbents [1-3]. The carbon shell encapsulating metallic core interferes in the electronic properties of the nanocapsules (NCs) through changing the ratio of $\mathrm{sp}^{2}$ to $\mathrm{sp}^{3}$ of the carbonaceous species. It is confirmed that the single-walled carbon nanotubes
(SWCNTs) or their bundles show the classical transport properties such as Coulomb blockade, energy quantization, Luttinger liquid characteristics and ballistic transport [4,5]. It is usually difficult for the multi-walled carbon nanotubes (MWCNTs) to make electrical contact between neighbor graphite layers because the total conductance is significantly limited by the charge carrier transport [6]. Variable range hopping (VRH) conduction, weak localization, resonant tunneling phenomena, universal conductance fluctuations, or Aharanov-Bohm oscillations of magnetoresistance, may appear or become dominant in the electrical transport of MWCNTscontaining systems $[7,8]$.

$\mathrm{Sn}$ is a typical metal with low melting point, high electrical conductivity, superconductivity, electrochemical activity and favorable behaviors. Bulk Sn has a relatively long coherence length of $\xi(0)(200 \mathrm{~nm})$ [9] at nanoscale. In the range of the coherence length $(0-200 \mathrm{~nm})$, the superconductive behaviors are expected to be significantly altered, for examples, a noticeable change in superconducting transition temperature $\left(T_{C}\right)$, decreased penetration depth, an enhancement in zero-field critical temperature, and finite residual resistance $[10,11]$. Coexistence of ferromagnetism and superconductivity was also found in Sn nanoparticles (NPs) with the size in range of $9-16 \mathrm{~nm}$ [12]. One-dimensional (1D) monocrystal $\mathrm{Sn}$ nanowires show $T_{\mathrm{C}}$ close to $3.7 \mathrm{~K}$; however both the electrical transport and the critical field are greatly size-related [13]. It has been reported that the superconductivity of carbon-coated Sn@C NCs will be

\footnotetext{
${ }^{1}$ Key Laboratory of Materials Modification by Laser, Ion and Electron Beams (Ministry of Education (MoE)), School of Materials Science and Engineering, Dalian University of Technology, Dalian 116024, China

${ }^{2}$ Shenyang National Laboratory for Materials Science, Institute of Metal Research, International Centre for Materials Physics, Chinese Academy of Sciences, Shenyang 110016, China

${ }^{3}$ Key Laboratory for Anisotropy and Texture of Materials (MoE), School of Materials and Engineering, Northeastern University, Shenyang 110819, China

*Corresponding authors (emails: dongxl@dlut.edu.cn (Dong X); zdzhang@imr.ac.cn (Zhang Z))
} 
destroyed as the size decreases down to $40 \mathrm{~nm}$. However, it has also been demonstrated for the carbon-coated $\mathrm{Sn}$ nanorods with diameter of $50 \mathrm{~nm}$ and length of $200 \mathrm{~nm}$, the critical magnetic field is almost 25 times higher than that of bulk Sn [14]. Large electrical current was also detected for MWCNTs encapsulated Sn nanowire, which can raise a local heating and in turn suppresses the superconductivity [15].

Electrical conduction in a nanocomposite consisting of the conductive and the insulative phases is usually attributed to electrical network and the percolation, in which the continuous conducting network or tunneling between isolated conducting particles would be concomitant [16]. A finite conductivity is ascribed to the inter-particles tunneling through the dielectric regime in the absence of metallic continuum. Kubo and co-workers [17] revealed that the energy gap between the nearest neighboring energy level increased rapidly with reducing the sizes of metal particles, and thus their physical properties differ from those of bulk metals. If the discrete gap becomes larger than the thermal energy $k_{\mathrm{B}} T$, a pronounced quantum size effect will happen. Meanwhile, the surface effect plays a prominent role in the conductivity. For a random mixture of granular superconducting/insulating phases, the grains would be weakly coupled by Josephson junction separating two superconductors by an insulating thin film [18], when the charging energy could not be neglected. The conductivity of such composite would be driven by a competition between the charging energy and Josephson energy. Maximum supercurrent $I_{\mathrm{C}}$ of Josephson junction is the critical current and can be easily observed as Josephson energy is far greater than the charging energy, which would be characteristic of superconductivity for the systems [19].

Up to now, carbon-coated Sn NPs have been fabricated by multifarious strategies, most of which are chemically prepared such as by aerosol spray pyrolysis technique, thermolysis of allyltriphenyltin, chemical vapor deposition [20-22]. In this work, a facile method of direct current (DC) arc-discharge plasma process is applied in the synthesis of CNTs encapsulated monocrystalline Sn nanorods (Sn@CNT NRs). It is a conventional route with merits of in situ preparation, variable nanostructures, large production yield, nonpollution and feasibility in operation. The electronic transport behaviors of Sn@CNT NRs are investigated to gain an insight into contributions from the carbonaceous shell and the superconductive Sn core. This preparation way could result in the high purity of nanocomposites and the well-defined junctions between Sn@CNT NRs.

\section{EXPERIMENTAL SECTION}

\section{Synthesis}

The MWCNTs encapsulated Sn nanorods were prepared by a conventional arc-discharge plasma method [23]. Raw target was a bulk metal Sn (99.99\% in purity) as the anode, while a carbon rod was used as the cathode. At $10^{-2} \mathrm{~Pa}$, the methane gas $(0.01 \mathrm{MPa})$ and argon gas $(0.02 \mathrm{MPa})$ were filled to the work chamber as the atmosphere. The distance between two electrodes was controlled in range of 3-4 $\mathrm{mm}$. The arc voltage was maintained at $\sim 20 \mathrm{~V}$, and meanwhile the current was kept at $90 \mathrm{~A}$. After more than $12 \mathrm{~h}$, the nanopowder products were then collected from the water-cooled wall of the work chamber. The nucleation and growth steps for Sn@CNT NRs were discussed in details in our previous publication [23].

\section{Characterization}

The phases of the as-prepared Sn@CNT NRs were confirmed by X-ray diffraction (XRD, PANalytical Empyrean) using $\mathrm{Cu}$ Ka radiation $(\lambda=1.5416 \AA)$. Morphologies, interfaces and crystallographic details were characterized by scanning transmission electron microscopy (STEM) (NOVA NanoSEM 450, $300 \mathrm{keV}$ ). Raman spectrum was recorded using the optical source of a laser with excitation wavelength of $632.8 \mathrm{~nm}$. X-ray photoelectron spectroscopy (XPS, Thermo Escalab 250Xi) utilizing monochromatic $\mathrm{Al} \mathrm{Ka}(h v=1486.6 \mathrm{eV})$ radiation as theta probe was adopted to analyze the surface species on Sn@CNT NRs. For electrical resistivity measurement, the as-prepared Sn@CNT NRs powder was pressed into a slice under $20 \mathrm{MPa}$ in a steel die, and cut into a size of $10 \times 10 \mathrm{~mm}^{2}$. This superconducting composite sheet has a density of $5.93 \mathrm{~g} \mathrm{~cm}^{-3}$. The temperature-dependent resistivity of Sn@CNT NRs composite was measured on physical property measurement system (PPMS, Quantum Design).

\section{RESULTS AND DISCUSSION}

\section{Structure, morphology and surface of Sn@CNT NRs}

As shown in Fig. 1, the XRD pattern of the as-prepared Sn@CNT NRs consists of a tetragonal $\beta$-Sn phase (JCPDS No. 01-086-2265) with the diffraction peaks of (200), (101) and (211) planes. The mean crystal size of Sn cores is estimated by Scherer equation: $D_{c}=0.89 \lambda /(B \cdot \cos \theta)$, where $D_{c}$ is the grain size, $B$ is the full width at half maximum (FWHM) of diffraction peak, $\theta$ is the diffraction angle, and $\lambda$ is X-ray wavelength $(1.5418 \AA)$. Ac- 


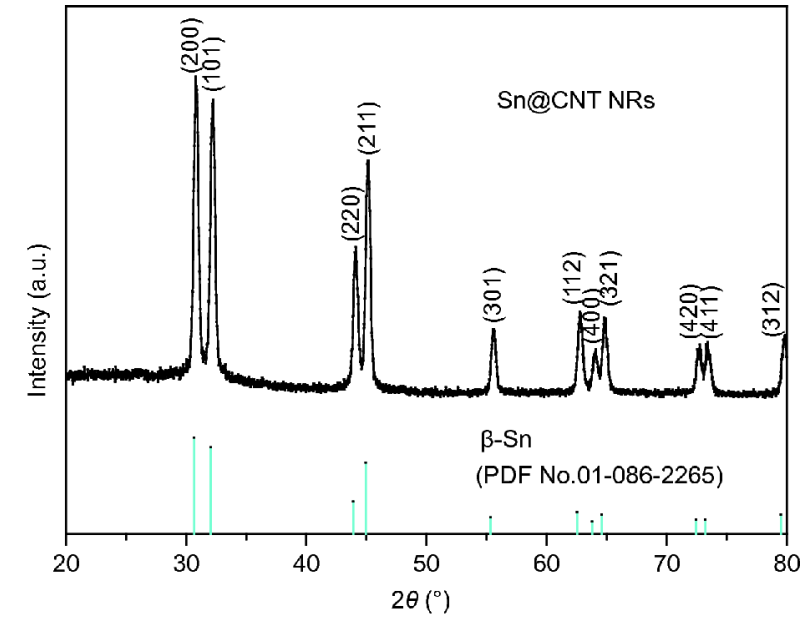

Figure 1 XRD pattern of the as-prepared powder of Sn@CNT NRs.

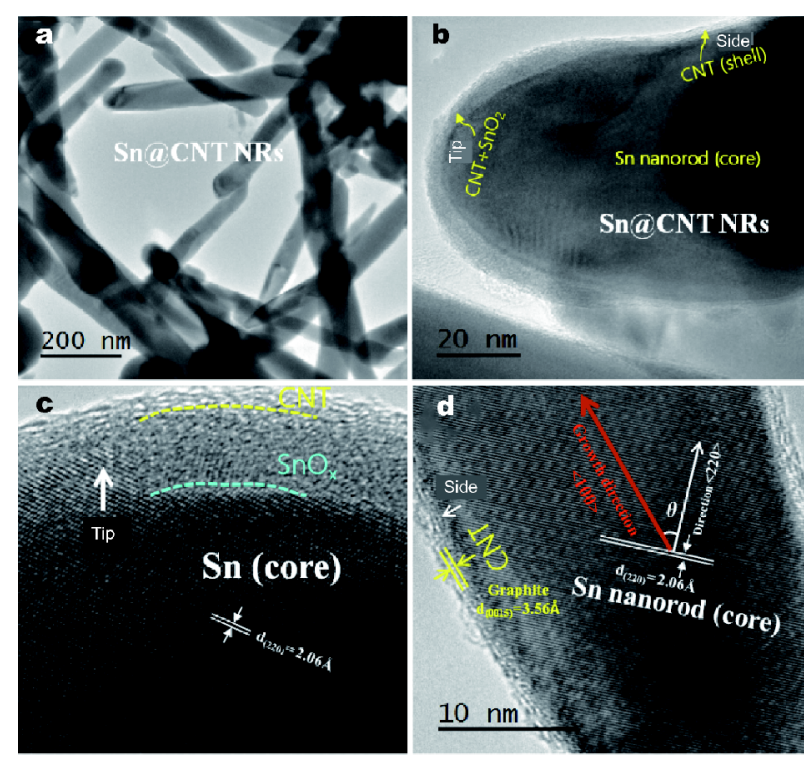

Figure 2 HRTEM images of the as-prepared Sn@CNT NRs.

cordingly, the calculated grain size of Sn core along $<220>$ crystallographic direction is about $72 \mathrm{~nm}$. There is no diffraction peaks of CNTs in XRD pattern, ascribed to the thin thickness of graphite shell, but well been displayed in high resolution TEM (HRTEM) pictures (Fig. $2 c, d)$. No stannic oxides emerge in XRD profile, but have been observed at the tip region of Sn@CNT NRs (Fig. 2c) and detected by XPS (Fig. 3b, c).

As shown in Fig. 2, the Sn@CNT NRs are 1D rod-like nanostructures with about $40-90 \mathrm{~nm}$ in diameter and 400-500 nm in length. The core is composed of a crystal Sn nanorod, which is completely encapsulated by CNTs.
At the tip sites of Sn@CNT NRs, the shell presents dual interfacial configuration consisting of CNTs outer layer and $\mathrm{SnO}_{x}$ inner layer, as shown in Fig. 2b. The detailed structure further reveals that the inner layer of $\mathrm{SnO}_{x}$ is made of the epitaxially grown oxide along certain lattice of $\mathrm{Sn}$ core and a random stacking of amorphous oxide (Fig. 2c). Existence of $\mathrm{SnO}_{x}$ layer about $5-8 \mathrm{~nm}$ in thickness predicates higher activity of the tip region of Sn@CNT NRs, which has formed in the passivation process. CNTs outer shell with $2-3 \mathrm{~nm}$ in thickness consists of 3-4 graphene layers, verified from the interplanar spacing of $3.56 \AA$ ((0015) lattice planes of graphite) [23]. Although a substantial distortion of CNTs shell appears at the tip region, the integrity of CNTs has been well retained. Monocrystalline $\mathrm{Sn}$ nanorod is well exhibited by one lattice image of (220) planes with $2.06 \AA$ of interplanar spacing and the crystal growth direction of [100], as shown in Fig. 2c, d. Crystallographic angle between the growth direction and [220] is $45^{\circ}$. The favorable growth direction of 1D Sn@CNT NRs will be decided mainly by the energy conditions for one anisotropic crystallite. It is considered that the surface free energy of a crystallographic plane $(h k l)$ may be expressed as $G_{h k l}=$ $\mu_{i} \times n_{h k l}$, where $\mu_{i}$ is the chemical potential of the species and $n_{h k l}$ is the atom number located on the $(h k l)$ plane [24]. The crystallographic planes possessing relatively higher surface free energies are thermodynamically more unstable and vulnerable to the attack from external atoms, and thus possess faster growth rates than other lattice planes with lower surface free energies. In a tetragonal system of $\beta$-Sn, the (100) planes have the highest atom numbers per unit area (0.2695 atoms $\left.\AA^{-2}\right)$ [25] and the highest surface free energy. Namely, the Sn@CNT NRs have a preferential growth along [100] direction for 1D Sn nanorod. In addition, arc-discharge plasma can greatly affect the structural perfection of CNTs and result in a relatively large diameter distribution of Sn@CNT NRs. However, they would have less effect on the preferential growth direction of monocrystal Sn nanorod.

XPS measurements were carried out to identify chemical composition of Sn@CNT NRs. The survey spectrum of Fig. 3a indicates 8.86 wt.\% of O, 52.50 wt.\% of Sn and 38.64 wt.\% of $\mathrm{C}$, respectively. The oxygen is attributed to the adsorbed $-\mathrm{OH} / \mathrm{O}_{2}$ species in air and the $\mathrm{SnO}_{x}$ inner layer at the tip of Sn@CNT NRs. Fig. 3b-d show the detailed photoelectron core level spectra of $\mathrm{Sn}, \mathrm{O}$ and $\mathrm{C}$ elements. The peaks in Fig. $3 \mathrm{~b}$ correspond to the binding energies of $\mathrm{Sn} 3 \mathrm{~d}$ electrons with valances of 0 (pure $\mathrm{Sn}$, $484.40 \mathrm{eV}$ for $3 \mathrm{~d}_{5 / 2}, 492.86 \mathrm{eV}$ for $3 \mathrm{~d}_{3 / 2}$ electrons), +2 $\left(\mathrm{SnO}, 494.61 \mathrm{eV}\right.$ for $3 \mathrm{~d}_{3 / 2}$ electrons $)$ and $+4\left(\mathrm{SnO}_{2}\right.$, 

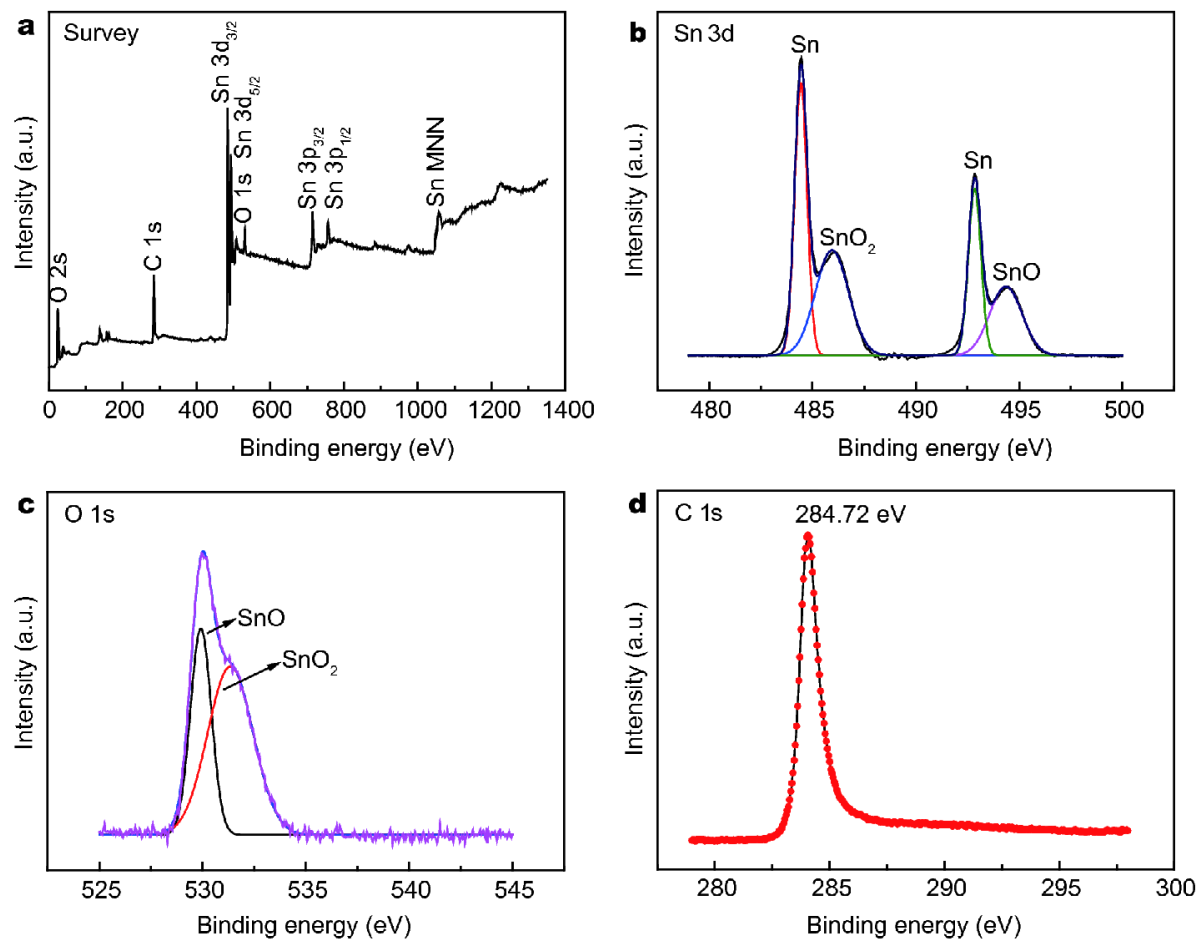

Figure 3 XPS spectra of the as-prepared powder of Sn@CNT NRs.

$486.20 \mathrm{eV}$ for $3 \mathrm{~d}_{5 / 2}$ electrons). In Fig. $3 \mathrm{c}$, the feature of $\mathrm{O} 1 \mathrm{~s}$ electrons at binding energies of 530.19 and $531.56 \mathrm{eV}$ are assigned to tin oxides of $\mathrm{SnO}$ and $\mathrm{SnO}_{2}$, well matching to those detected by Sn electrons. The surface graphitic carbon of Sn@CNT NRs is also confirmed by the binding energy of $\mathrm{C} 1 \mathrm{~s}$ electrons at 284.72 eV (Fig. 3d), which will be further supported by the Raman signal in the following section.

The carbon shell of Sn@CNT NRs is analyzed by Raman spectrum, as shown in Fig. 4. Two intensive peaks of D- and G-band are allocated at Raman shifts of about 1,335 and $1,570 \mathrm{~cm}^{-1}$, corresponding to $A_{1 \mathrm{~g}}$ and $\mathrm{E}_{2 \mathrm{~g}}$ vibration modes of carbon atoms, respectively [26]. G band usually indicates the original feature of graphite, while $\mathrm{D}$ band suggests a disordered carbonaceous structure [27]. Strong D-peak, normally aroused in the presence of inplane substitutional heteroatoms, vacancies, grain boundaries or other defects, indicates a large amount of disordered carbon existing in the CNT shell of Sn@CNT NRs, as observed in the HRTEM image of Fig. 2d. The Gband is typical for graphite and carbon blacks, originating from the stretching vibration of any $\mathrm{C}$ pairs of $\mathrm{sp}^{2}$ sites [28]. In the CNT shell, the in-plane correlation length $L_{\mathrm{a}}$ can be expressed by Tuinstra-Koenig equation $L_{\mathrm{a}}=C_{\lambda} \cdot\left(I_{\mathrm{D}} /\right.$ $\left.I_{\mathrm{G}}\right)^{-1}$, where $C_{\lambda}$ is about $4.4 \mathrm{~nm}$, the ratio of $\left(I_{\mathrm{D}} / I_{\mathrm{G}}\right)$ equals

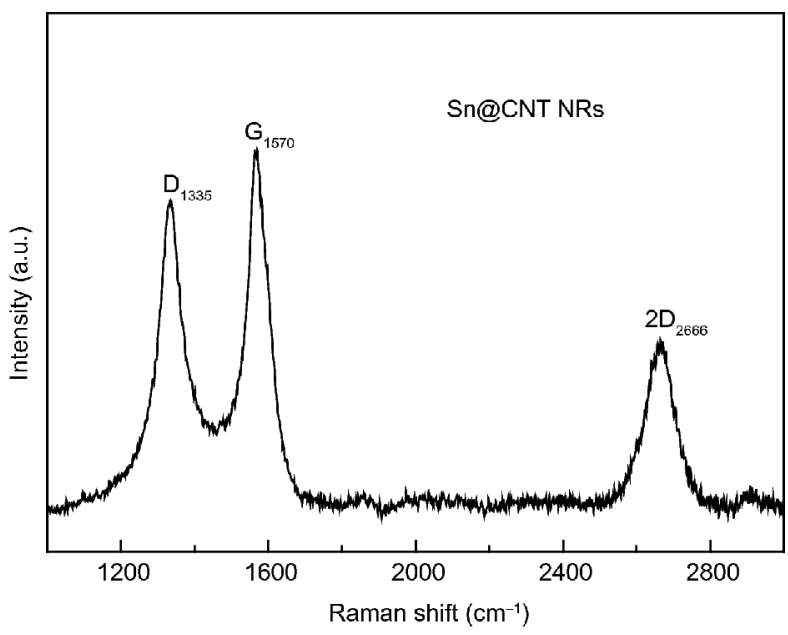

Figure 4 Raman spectrum of Sn@CNT NRs.

to 0.993 obtained from a Lorentz fitting on Raman spectrum of Sn@CNT NRs, and thus the corresponding $L_{\mathrm{a}}$ is calculated as $4.43 \mathrm{~nm}$, a little smaller than $4.73 \mathrm{~nm}$ of the correlation length of pure MWCNTs reported in our previous work [29]. The smaller value of $L_{\mathrm{a}}$ implies that the CNT shell of Sn@CNT NRs has been greatly influenced by the metallic Sn rod, due to the peculiar co- 

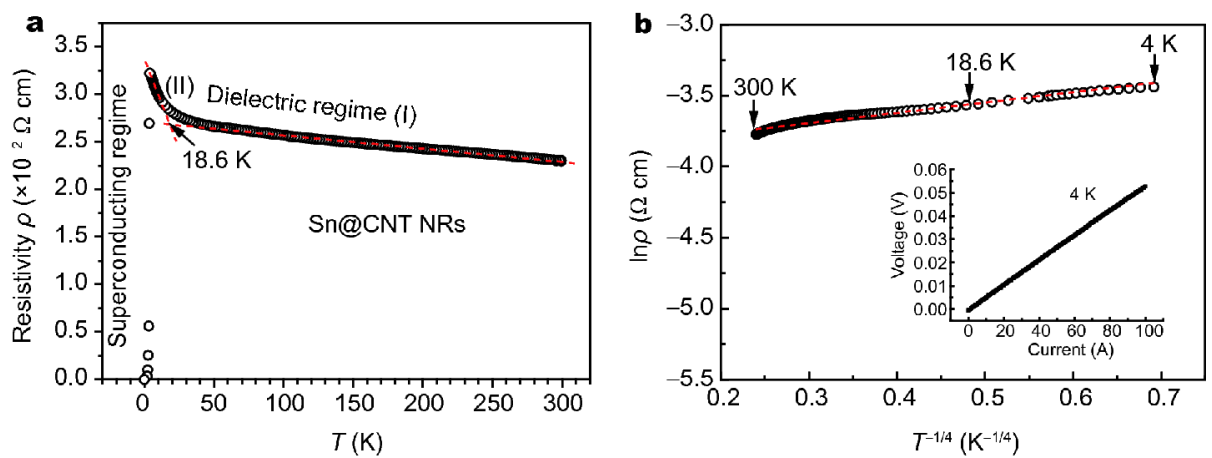

Figure 5 Temperature dependence of electric behaviors of Sn@CNT NRs sheet. (a) Resistivity $\rho$ vs. T; (b) logarithmic $\rho$ against $T^{-1 / 4}$.

evaporation process of arc-discharge plasma by which the well-developed CNT shell allowed the electrons from Sn atoms. Additionally, the peak denoted as $2 \mathrm{D}$ band at around $2,666 \mathrm{~cm}^{-1}$ is thought to originate from the finitesize crystals of graphite, which is an overtone of the $\mathrm{D}$ peak in the case of total lack of $c$-axis order.

\section{Electric transport of Sn@CNT NRs}

\section{Dielectric behavior above $T_{c}$}

Fig. 5 displays temperature dependent electric behaviors of the mechanically compressed Sn@CNT NRs sheet. Fig. 5 a distinctly shows that dielectric and superconductivity are separated at $3.7 \mathrm{~K}$. The dielectric regime contains two regions of (I) and (II), intergraded at $18.6 \mathrm{~K}$ with different slopes of the fitted lines. The similar behavior to pure CNTs suggests that the MWCNTs shell of Sn@CNT NRs dominates the dielectric performance. While the metallic Sn cores also promote the conductivity of Sn@CNT NRs sheet, judged by the resistivity ratio $\rho_{\mathrm{r}}=\rho_{(4 \mathrm{~K})} / \rho_{(300 \mathrm{~K})}$ $=3.2 \times 10^{-2} / 2.3 \times 10^{-2}=1.39$. The resistivity of Sn@CNT NRs is between the resistivity of the MWCNTs $(4.5 \Omega \mathrm{cm})$ [30] and bulk Sn $\left(1.1 \times 10^{-5} \Omega \mathrm{cm}\right)$ [31], indicating a great modification on the electric properties of pure CNTs by the metallic Sn component.

It had been proved that the semiconducting behavior of CNTs is decided by several factors, e.g., bond ratio of $\mathrm{sp}^{2}$ to $\mathrm{sp}^{3}, \pi$-orbitals in $\mathrm{sp}^{2}$, crystallization degree, structural perfection and morphology [32]. At a temperature above the transition point, electronic hopping is recognized as the main mechanism for CNTs and carbon fibers [31,33], during which the electrons are strongly confined within localized states and hop to the nearest neighbor as raising external temperature. Hopping conductivity happens when the Coulomb interaction of electrons is negligible and can be described by Mott-David (MD) VRH model
[7] $: \rho(T)=\rho_{0} \exp \left(T_{0} / T\right)^{1 / 4}$, where $\rho_{0}$ is a constant, $T_{0}$ is Mott characteristic temperature which can be obtained from $\ln \rho(T) \sim T^{-1 / 4}$ plot as drawn in Fig. 5b. It is revealed that a linear relation of $\ln \rho(T) \sim T^{-1 / 4}$ covers the whole temperature range of dielectric regions (I, II), from 300 to $4 \mathrm{~K}$ spanning the transition point of $18.6 \mathrm{~K}$, indicating one model of MD VRH is available to all dielectric behaviors of Sn@CNT NRs. For the pure CNTs, its dielectric behavior in the region II (below the transition point) is usually described by Shklovskii-Efros (SE) VRH model, i.e., $\ln \rho(T) \sim T^{-1 / 2}$ law, if a Coulomb gap is not negligible [33]. It is suggested that the dielectric difference between Sn@CNT NRs and pure CNTs is attributed to the conductive contribution from the metallic Sn core, that is to say, the Coulomb gap in the MWCNTs shell becomes weak in respect to the case of pure CNTs. Such phenomenon was also found in $\mathrm{NbC}(\mathrm{C})-\mathrm{C}$ nanocomposites, in which the metallic $\mathrm{NbC}$ nanocrystals play similar conductive contribution [34]. Coulomb gap $\Delta_{\mathrm{C}}=k_{\mathrm{B}} T_{0}$ [35], the slope of fitted line on resistivity curve as shown in Fig. 5b, was $0.021 \mathrm{meV}$ for present Sn@CNT NRs.

Negligible Coulomb interaction and weak Coulomb gap are remarkable for Sn@CNT NRs, which are attributed to the influence from metallic $\mathrm{Sn}$ core. Coulomb interactions open a soft Coulomb gap $\Delta_{\mathrm{C}}$ at Fermi level through the charging energy and may result in Coulomb blockade. As for Sn@CNT NRs, the linear $I-V$ relation at $4 \mathrm{~K}$ (as shown in the inset of Fig. 5b) is the evidence for weak Coulomb blockade in Sn@CNT NRs. Transition from the dielectric region I to II (Fig. 5a) essentially reflects an influence of thermal interference on the resistivity of Sn@CNT NRs. However, the thermal energy is unable to significantly influence the hopping conductivity in Sn@CNT NRs, and thus MD hopping mode is still compatible below the transition point of $18.6 \mathrm{~K}$. Tunneling effect is responsible for the phenomenon, gener- 

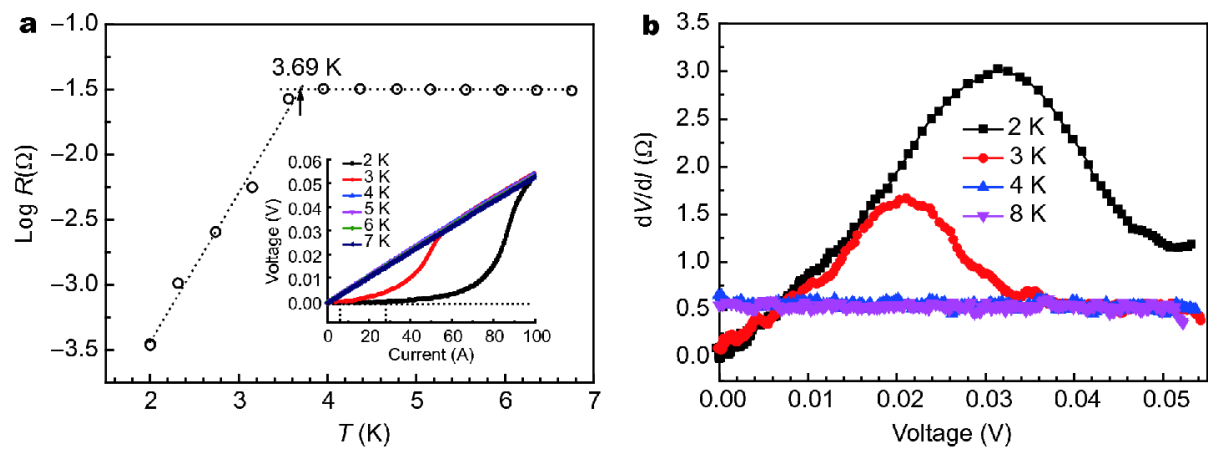

Figure 6 Superconducting behavior of Sn@CNT NRs. (a) Logarithmic resistance $R$ vs. temperature curve, the inset is $I-V$ characteristics at temperatures from 2 to $7 \mathrm{~K}$; (b) differential resistance $\mathrm{d} V / \mathrm{d} I$ as a function of bias voltage at temperatures from 2 to $8 \mathrm{~K}$.

ated through Josephson junction of Sn/CNT/Sn among the weakly linked Sn@CNT NRs. The coupling energy of $\mathrm{Sn} / \mathrm{CNT} / \mathrm{Sn}$ junction would favor the tunneling, if it governs the competition with the Coulomb charging energy.

Superconductivity of Sn@CNT NRs below $T_{C}$ The superconductivity of Sn@CNT NRs is illustrated by a sharp drop of resistivity to the minimum $\left(3.4 \times 10^{-4} \Omega \mathrm{cm}\right)$ (Figs 5a and 6a). The critical temperature $T_{\mathrm{C}}$ is determined as $3.69 \mathrm{~K}$, which is very close to $3.73 \mathrm{~K}$ of bulk Sn [23]. It has been demonstrated that a single-crystal Sn superconductor with size less than coherent length has usually no significant suppression at temperature of superconducting transition. That means the crystalline Sn nanorod core in Sn@CNT NRs solely determines the superconducting transition point, while the semiconductor-superconductor transition (SST) is ruled by core-shell structure in Sn@CNT NRs. Such a similar behavior was also observed in a graphene-metal hybrid [36] or a Josephson junction array [37]. For the present Sn@CNT NRs, Josephson junction of Sn/CNT/Sn layered structure and the weakly linked nanocapsules are essential in nanoscale [14].

The SST model for Sn@CNT NRs results from the competition between the charging energy $E_{\mathrm{C}}$ and Josephson coupling energy $E_{\mathrm{J}}$. The charging energy $E_{\mathrm{C}}=e^{2} / 2 C$, where $C$ is the inter-grain capacitance. $E_{\mathrm{C}}$ is the energy for accumulation of binding charges and can block a tunneling among Sn@CNT NRs. In compressed Sn@CNT NRs sheet, the contacting interface between two NCs can be considered as an equivalent plate capacitor consisted by two electrodes of metallic $\mathrm{Sn}$ nanorods and the dielectric medium of CNT. Its capacitance can be numerically estimated from equation $C=\varepsilon_{0} \varepsilon_{\mathrm{r}} D L / d$, where
$D$ and $L$ are the average diameter and length of one $\mathrm{Sn}$ nanorod, and $d$ is the thickness of double walls of CNT. Taking into account $\varepsilon_{\mathrm{r}}=2.5$ (carbon black), $D=50 \mathrm{~nm}$, $L=500 \mathrm{~nm}$ and $d=6 \mathrm{~nm}$, the capacitance is estimated as $C \approx 3.69 \times 10^{-17} \mathrm{~F}$, and thus the charging energy should be $E_{\mathrm{C}} \approx 3.46 \times 10^{-22} \mathrm{~J} . \quad E_{\mathrm{C}}$ can suppress the electron-pair tunneling through Josephson junction. Josephson coupling energy $E_{\mathrm{J}}$ is temperature dependent and given by $E_{\mathrm{J}}(T)=\frac{h}{8 e^{2}} \frac{\Delta(T)}{R_{\mathrm{N}}} \tanh \left(\frac{\Delta(T)}{2 k_{\mathrm{B}} T}\right)[38]$, where $h$ is Planck constant, $\Delta(T)$ is the temperature-dependent energy gap of each superconductor, $R_{\mathrm{N}}$ is the normal state resistivity of junction. $E_{\mathrm{J}}$ is also obtained from $E_{\mathrm{J}}(T)=(h / 4 \pi e) I_{\mathrm{C}}$ [39], where $I_{\mathrm{C}}$ is the critical current. From the inset of Fig. $6 \mathrm{a}$, $I-V$ curves illustrate the critical currents (also called Josephson supercurrent) at different temperatures, i.e., $I_{\mathrm{C}} \approx 4 \mathrm{~mA}$ at $3 \mathrm{~K}$ and $I_{\mathrm{C}} \approx 24 \mathrm{~mA}$ at $2 \mathrm{~K}$. Accordingly, Josephson coupling energies are calculated as $E_{\mathrm{J}} \approx 2.74$ $\times 10^{-19} \mathrm{~J}$ at $3 \mathrm{~K}$ and $1.64 \times 10^{-18} \mathrm{~J}$ at $2 \mathrm{~K}$. By comparison of $E_{\mathrm{J}}$ and $E_{\mathrm{C}}$, Josephson coupling energy is larger than the charging energy by 3-4 orders of magnitude, which can drive the electron-pair tunneling below $T_{\mathrm{C}}$. It has been indicated that if the Josephson coupling dominates over the charging energy, the system would be transformed to the superconducting phase [40]. The SST in these Sn@CNT NRs, as a typical disordered-system, is also similar to the phase-locking transition due to the percolation phenomena [38,41]. In Fig. 6a, a broad superconducting transition tail of about $1.7 \mathrm{~K}$ is found and can be described by the 'inverse Arrennius' law: $R(T)=R_{0} e^{T / T_{0}}$ [42]. The slope of logarithmic $R$ vs. $T$ curve, $1 / T_{0}$, approximately equal to $1 / C^{*} R_{\mathrm{N}}$, where $C^{*}$ is a constant and $R_{\mathrm{N}}$ is the normal state resistance of junction. It means the transition tail only depends on the normal state resistance of $\mathrm{Sn} / \mathrm{CNT} / \mathrm{Sn}$ junction, which would be determined by 
the geometrical arrangement of Sn@CNT NRs, i.e., the density, inter-NC spacing, morphology configuration and the tunneling percolation network. The superconducting transition tail of Sn@CNT NRs is quite different from the cases for 1D Sn nanowires [13], due to the unique CNTcoated core/shell structure.

In Fig. $6 \mathrm{~b}$, the differential resistance $\mathrm{d} V / \mathrm{d} I$ as a function of the bias voltage is plotted at temperatures from 2 to $8 \mathrm{~K}$. The distinct peaks at 2 and $3 \mathrm{~K}$, are recognized as the result from Andreev reflection under a superconducting state $[43,44]$, in which electrons/holes will be reflected from the interfaces of junction. The Andreev reflection and resultant excess conductance originates from the interfaces in Sn/CNT/Sn junctions of Sn@CNT NRs. Above $T_{C}$, the relation of $\mathrm{d} V / \mathrm{d} I$ vs. bias voltage obeys the normal ohm law and $R_{\mathrm{N}} \approx 0.5 \Omega$ can be obtained. By using the largest $I_{\mathrm{C}} \approx 24 \mathrm{~mA}$ at $2 \mathrm{~K}$, the $I_{\mathrm{C}} R_{\mathrm{N}}$ product $[45,46]$, a characteristic junction parameter, is $\sim 12 \mathrm{mV}$ for $\mathrm{Sn} / \mathrm{CNT} /$ Sn junction in Sn@CNT NRs.

In general, the electronic transport behavior of Sn@CNT NRs prepared by arc-discharge plasma here is characteristic of a transition from electronic hopping conduction to the electron-pair tunneling at $3.69 \mathrm{~K}$, which is quite different from the transition of normal metal to the superconductor [15,21,23]. Above results supply crucial data for novel superconducting nanostructures, and may serve as the key parts of a phase-based switching, sensors and electronic devices, combining the tunable function of semiconductor with the long-range coherence of superconductor.

\section{CONCLUSIONS}

The MWCNTs encapsulated Sn nanorods (Sn@CNT NRs) were prepared by a DC arc-discharge plasma process in methane $\left(\mathrm{CH}_{4}\right)$ atmosphere. These Sn@CNT NRs are typical $40-90 \mathrm{~nm}$ in diameter and $400-500 \mathrm{~nm}$ in length. The monocrystal $\mathrm{Sn}$ rods result from anisotropic growth along [100] crystallographic direction of $\beta-S n$ lattice. Dielectric behavior of Sn@CNT NRs follows MD VRH $\left[\ln \rho(T) \propto T^{-1 / 4}\right]$ model above the superconducting transition temperature of $3.69 \mathrm{~K}$, implying that a great modification was imposed onto MWCNTs shell by the metallic Sn core and further confirmed with the resistivity ratio $\rho_{r}\left(\rho_{(4 \mathrm{~K})} / \rho_{(300 \mathrm{~K})}\right)$ of 1.39 . Josephson junction of $\mathrm{Sn} /$ $\mathrm{CNT} / \mathrm{Sn}$ layered structure is assembled in the compressed Sn@CNT NRs sheet and is mainly responsible for the superconducting behavior. Josephson coupling energy is estimated as $2.74 \times 10^{-19} \mathrm{~J}$ (at $3 \mathrm{~K}$ ), far larger than $3.46 \times 10^{-22} \mathrm{~J}$ of the charging energy for Sn@CNT NRs. A broad tail of superconducting transition is the char- acteristic of Sn@CNT NRs, determined by the normal state resistance $R_{\mathrm{N}}$ of $\mathrm{Sn} / \mathrm{CNT} / \mathrm{Sn}$ junction (calculated as $0.5 \Omega$ ). The measured critical currents (Josephson supercurrent) of Sn@CNT NRs sheet are $4 \mathrm{~mA}$ (at $3 \mathrm{~K}$ ) and $24 \mathrm{~mA}$ (at $2 \mathrm{~K}$ ).

Received 22 January 2018; accepted 10 April 2018; published online 7 May 2018

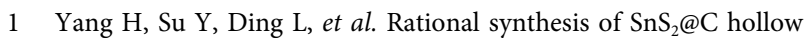
microspheres with superior stability for lithium-ion batteries. Sci China Mater, 2017, 60: 955-962

2 Zhang L, Ding Y, Li R, et al. Ni-Based metal-organic framework derived $\mathrm{Ni@C} \mathrm{nanosheets} \mathrm{on} \mathrm{a} \mathrm{Ni}$ foam substrate as a supersensitive non-enzymatic glucose sensor. J Mater Chem B, 2017, 5: 5549-5555

3 Shah A, Ding A, Wang Y, et al. Enhanced microwave absorption by arrayed carbon fibers and gradient dispersion of Fe nanoparticles in epoxy resin composites. Carbon, 2016, 96: 987-997

4 Tans SJ, Devoret MH, Dai H, et al. Individual single-wall carbon nanotubes as quantum wires. Nature, 1997, 386: 474-477

5 Krstić V, Roth S, Burghard M. Phase breaking in three-terminal contacted single-walled carbon nanotube bundles. Phys Rev B, 2000, 62: R16353-R16355

6 Poncharal P, Frank S, Wang ZL, et al. Conductance quantization in multiwalled carbon nanotubes. Eur Phys J D, 1999, 9: 77-79

7 Mott NF, David EA. Electronic Processes in Noncrystalline Materials, 2nd ed. Oxford: Oxford University Press, 1979, pp55

8 Webb RA, Washburn S, Umbach CP, et al. Observation of h/e Aharonov-Bohm oscillations in normal-metal rings. Phys Rev Lett, 1985, 54: 2696-2699

9 Tian M, Wang J, Kurtz JS, et al. Dissipation in quasi-one-dimensional superconducting single-crystal Sn nanowires. Phys Rev B, 2005, 71: 104521

10 Liu Y, Zadorozhny Y, Rosario MM, et al. Destruction of the global phase coherence in ultrathin, doubly connected superconducting cylinders. Science, 2001, 294: 2332-2334

11 Lau CN, Markovic N, Bockrath M, et al. Quantum phase slips in superconducting nanowires. Phys Rev Lett, 2001, 87: 217003

12 Li WH, Wang CW, Li CY, et al. Coexistence of ferromagnetism and superconductivity in Sn nanoparticles. Phys Rev B, 2008, 77: 094508

13 Tian M, Wang J, Snyder J, et al. Synthesis and characterization of superconducting single-crystal Sn nanowires. Appl Phys Lett, 2003, 83: $1620-1622$

14 Wang ZH, Geng DY, Han Z, et al. Large critical magnetic field and tunneling anomaly behavior of superconducting carbon-coated $\mathrm{Sn}$ nanorods and nanoparticles. J Appl Phys, 2010, 108: 013903

15 Tombros N, Buit L, Arfaoui I, et al. Charge transport in a single superconducting tin nanowire encapsulated in a multiwalled carbon nanotube. Nano Lett, 2008, 8: 3060-3064

16 Sheng P, Sichel EK, Gittleman JI. Fluctuation-induced tunneling conduction in carbon-polyvinylchloride composites. Phys Rev Lett, 1978, 40: 1197-1200

17 Kubo R, Kawabata A, Kobayashi S. Electronic properties of small particles. Annu Rev Mater Sci, 1984, 14: 49-66

18 Entin-Wohlman O, Kapitulnik A, Shapira Y. Dependence of $T_{\mathrm{c}}$ on the normal-state resistivity in granular superconductors. Phys Rev 


\section{B, 1981, 24: 6464-6468}

19 Steinbach A, Joyez P, Cottet A, et al. Direct measurement of the Josephson supercurrent in an ultrasmall Josephson junction. Phys Rev Lett, 2001, 87: 137003

20 Jankovic L, Gournis D, Trikalitis PN, et al. Carbon nanotubes encapsulating superconducting single-crystalline tin nanowires. Nano Lett, 2006, 6: 1131-1135

21 Zou Y, Wang Y. Sn@CNT nanostructures rooted in graphene with high and fast Li-storage capacities. ACS Nano, 2011, 5: 8108-8114

22 Pol VG, Thiyagarajan P, Acharya S, et al. Superconducting nanocrystalline tin protected by carbon. Langmuir, 2009, 25: 2582-2584

23 Liu C, Huang H, Cao G, et al. Enhanced electrochemical stability of Sn-carbon nanotube nanocapsules as lithium-ion battery anode. Electrochim Acta, 2014, 144: 376-382

24 Tiller WA. The Science of Crystallization: Microscopic Interfacial Phenomena. New York: Cambridge University Press, 1991

25 Hsu YJ, Lu SY. Vapor-solid growth of Sn nanowires: growth mechanism and superconductivity. J Phys Chem B, 2005, 109: 4398-4403

26 Marcinek M, Hardwick LJ, Richardson TJ, et al. Microwave plasma chemical vapor deposition of nano-structured $\mathrm{Sn} / \mathrm{C}$ composite thin-film anodes for Li-ion batteries. J Power Sources, 2007, 173: 965-971

27 Obraztsova ED, Fujii M, Hayashi S, et al. Raman identification of onion-like carbon. Carbon, 1998, 36: 821-826

28 Carlo Ferrari A, Robertson J. Raman spectroscopy of amorphous, nanostructured, diamond-like carbon, and nanodiamond. Philos Trans R Soc A-Math Phys Eng Sci, 2004, 362: 2477-2512

29 Lu B, Huang H, Dong XL, et al. Catalytic pyrogenation synthesis of $\mathrm{C} / \mathrm{Ni}$ composite nanoparticles: controllable carbon structures and high permittivities. J Phys D-Appl Phys, 2010, 43: 105403-105409

30 Kittel C. Introduction to Solid State Physics 8th Edition. New York: Wiley, 2005, pp107

31 Yosida Y, Oguro I. Variable range hopping conduction in multiwalled carbon nanotubes. J Appl Phys, 1998, 83: 4985-4987

32 Chen CW, Robertson J. Nature of disorder and localization in amorphous carbon. J Non-Crystalline Solids, 1998, 227-230: 602606

33 Shen J, Chen Z, Wang N, et al. Electrical properties of a single microcoiled carbon fiber. Appl Phys Lett, 2006, 89: 153132

34 Li D, Li WF, Ma S, et al. Electronic transport properties of $\mathrm{NbC}$ (C)-C nanocomposites. Phys Rev B, 2006, 73: 193402

35 Benoit JM, Corraze B, Chauvet O. Localization, Coulomb inter- actions, and electrical heating in single-wall carbon nanotubes/ polymer composites. Phys Rev B, 2002, 65: 241405

36 Allain A, Han Z, Bouchiat V. Electrical control of the superconducting-to-insulating transition in graphene-metal hybrids. Nat Mater, 2012, 11: 590-594

37 van der Zant HSJ, Elion WJ, Geerligs LJ, et al. Quantum phase transitions in two dimensions: Experiments in Josephson-junction arrays. Phys Rev B, 1996, 54: 10081-10093

38 Imry Y, Strongin M. Destruction of superconductivity in granular and highly disordered metals. Phys Rev B, 1981, 24: 6353-6360

39 Feigel'Man MV, Skvortsov MA, Tikhonov KS. Proximity-induced superconductivity in graphene. J Exp Thero Phys, 2008, 88: 747751

40 Sondhi SL, Girvin SM, Carini JP, et al. Continuous quantum phase transitions. Rev Mod Phys, 1997, 69: 315-333

41 Deutscher G, Imry Y, Gunther L. Superconducting phase transitions in granular systems. Phys Rev B, 1974, 10: 4598-4606

42 Frydman A, Naaman O, Dynes RC. Universal transport in twodimensional granular superconductors. Phys Rev B, 2002, 66: 340351

43 Aminov BA, Golubov AA, Kupriyanov MY. Quasiparticle current in ballistic constrictions with finite transparencies of interfaces. Phys Rev B, 1996, 53: 365-373

44 Gül Ö, Günel HY, Lüth H, et al. Giant magnetoconductance oscillations in hybrid superconductor-semiconductor core/shell nanowire devices. Nano Lett, 2014, 14: 6269-6274

45 Oostinga JB, Maier L, Schüffelgen $\mathrm{P}$, et al. Josephson supercurrent through the topological surface states of strained bulk HgTe. Phys Rev X, 2013, 3: 021007

46 Liu G, Zhang Y, Lau CN. Gate-tunable dissipation and "superconductor-insulator" transition in carbon nanotube Josephson junctions. Phys Rev Lett, 2009, 102: 016803

Acknowledgements This work was financially supported by the National Natural Science Foundation of China (51331006 and 51271044).

Author contributions Wang D, Li D, Muhammad J, Zhang X, Dong X and Zhang Z designed the experiments; Wang D, Zhou Y, Wang Z, Lu S performed the experiments; Wang D, Li D, Zhang $X$ analyzed the data; Wang $\mathrm{D}$, Dong $\mathrm{X}$ and Zhang $\mathrm{Z}$ wrote the paper; all authors discussed the results and commented on the manuscript.

Conflict of interest The authors declare no conflict of interest. 


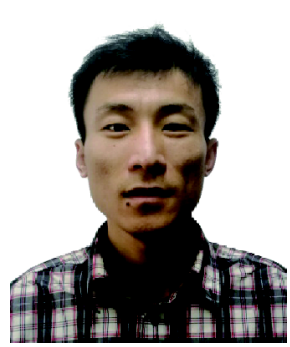

Dongxing Wang is currently a PhD student under the supervision of Prof. Xinglong Dong at the School of Materials Science and Engineering in Dalian University of Technology. His current research focuses on the fabrication, electronic properties and application of core-shell nanoparticles.

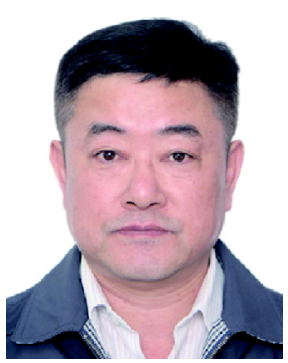

Xinglong Dong is a professor of Dalian University of Technology. He obtained his $\mathrm{PhD}$ at the Institute of Metal Research, Chinese Academy of Sciences. His research work focuses on the synthesis and characterization of core-shell nanoparticles, as well as the magnetic, electric, electromagnetic and electrochemical properties of nanocomposites.

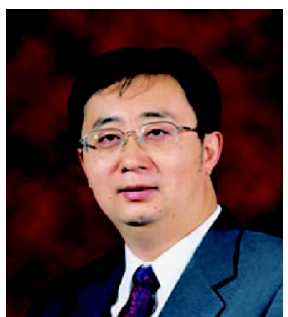

Zhidong Zhang is currently the director of the Magnetism and Magnetic Materials Division, Shenyang National Laboratory for Materials Science. He obtained his PhD at the Institute of Metal Research, Chinese Academy of Sciences in 1989. His work involves in the spin wave theory, new rare-earth transition-metal compounds, nanocomposite magnetic materials, magnetic phase transitions and applications of magnetic materials.

\section{原位热等离子体法组建碳纳米管封装锡纳米棒及其电子输运行为}

王东星 ${ }^{1}$, 李达 ${ }^{2}$, 贾伟迪 ${ }^{1}$, 周远良 ${ }^{1}$, 张雪峰 ${ }^{3}$, 王子明 ${ }^{1}$, 陆珊珊 ${ }^{1}$, 董星龙 ${ }^{1 *}$, 张志东 ${ }^{2^{*}}$

摘要 通过简易的电弧放电等离子法, 用块体锡作为原料靶材、甲烷作为气态碳源组装了多壁碳纳米管封装单晶锡纳米棒(Sn@CNT NRs). Sn@CNT NRs直径为40-90 nm, 长为400-500 nm. 碳纳米管保护了内部的锡纳米棒免于氧化, 并且构建了一种结合良好的纳米复合 材料. 温度相关的 $I-V$ 曲线及电阻率的测量显示, 在超导临界温度 $3.69 \mathrm{~K}$ 之上 $\mathrm{Sn} @ \mathrm{CNT}$ NRs的介电行为归因于碳纳米管壳, 且按照莫特-戴 维变程跳跃 $\left(\ln R(T) \propto T^{-1 / 4}\right)$ 模式, 半导体-超导体的转变 $(\mathrm{SST})$ 是Sn@CNT纳米棒的一个显著特征. 此外, 发现Sn@CNT NRs的超导行为是由 锡/碳纳米管/锡的层结构形成的约瑟夫森结导致的. 